\title{
Advanced Design of a Low Energy Electron Source
}

\author{
Alberto Leggieri ${ }^{1}$, Davide Passi ${ }^{1}$, Franco Di Paolo ${ }^{1}$, Bruno Spataro ${ }^{2}$ and Egor Dyunin ${ }^{3}$ \\ ${ }^{1}$ Dept. of Electronic Engineering, University of Rome "Tor Vergata", Via del Politecnico 1, 00133, Roma, Italy \\ ${ }^{2}$ Istituto Nazionale di Fisica Nucleare - Laboratori Nazionali di Frascati, Via E. Fermi 40, 00044 Frascati (Roma), Italy \\ ${ }^{3}$ Ariel University, Kiriyat Ha-Mada, 40700, Ariel, Israel \\ alberto.leggieri@uniroma2.it
}

\begin{abstract}
A multiphysics-based modeling design of a low energy electron source using a thermionic cathode is described in this paper. The proposed device produces a narrow beam employable in delicate applications where dimensions are critical. The effects of multiple physics influencing factors due to the cathode heating over the beam dynamics have been predicted through a multiphysics design approach. This paper would provide the needed knowledge for virtual prototyping of such devices. For this aim, several strategies have been adopted to obtain a simple model, which shows clearly the investigated mechanisms. According to this study, the appropriate materials and shapes can be chosen.
\end{abstract}

Index Terms - Multi-physics modeling, FEM methods, Computational Electromagnetics, Electron Gun.

\section{INTRODUCTION}

$\mathrm{L}$ ow energy electron source are devices which produce a electron streams with a typical energy obtainable with electrostatic acceleration. Such devices, usually called Electron Guns (e-Gun's), are employed in vacuum tubes to produce the main electron current for generating output signals [1]. In many applications, very small beam dimension are required, as sub-millimeter waves [2-3], electromagnetic environmental instrumentation and electron microscopes for spatial applications [4-6]. For such devices, a thermionic filament is desirable for several reasons: It requires a high vacuum of about $10^{-7}$ Torr; it is not very expensive and robust [5].

However in thermionic devices, the power dissipation of the cathode produces a considerable temperature increase and induces a thermal expansion of both the cathode and the anode, which is warmed by the heat transfer operated by the non-ideal vacuum between anode and cathode regions. In this range of small dimensions, an uncontrolled thermal expansion may produce destructive effects over the desired beam dynamics.

This study proposes the design of an electron gun based on a Multiphysics modeling approach in order to prevent the undesired behavior. An e-Gun designed by following such criterion can be also connected to micro vacuum tubes and, implemented with an opportune heat exchanger. Many aspects have been investigated at the same time such as mechanical stress or thermal expansion together with the behavior of charged particles immerged in electric fields. By a ThermoMechanical (TM) stationary analysis, temperature and deformation have been determined when the heat generated by the cathode has been diffused on all the reachable e-Gun components. On the deformed geometry the particle trajectory has been computed. Since the Electrostatic (ES) fields are altered by the particles presence, the problem has been solved for the particles and fields simultaneously by coupling a Charge Particle Tracing (CPT) and an ES analysis [7].

\section{ElECTRON Gun DESIGN AND MultiPhySICS MODEL}

The e-Gun has been designed by following the theory of electron beam design reported in [1]. The beam dimensions are consistent with vacuum devices already studied in the $\mathrm{THz}$ frequency range [8]. In the proposed computational model, a drift tube has been added to the geometry, in order to evaluate the beam properties. Anode and cathode are made of molybdenum; the interaction region is made of non-ideal vacuum which is air at the pressure of $10^{-7}$ bar. In order to ensure the vacuum sealing, an alumina insulator is present. A ceramic shell supports the heater, connected to the tungsten emitting surface which overlooks to the vacuum interaction region. Between the heater and the cathode structure, a vacuum gap of $30 \mu \mathrm{m}$ thickness has been inserted to avoid electrical and thermal contact. The spacing between anode and cathode is $4.0 \mathrm{~mm}$. The geometry is depicted in Figure 1.

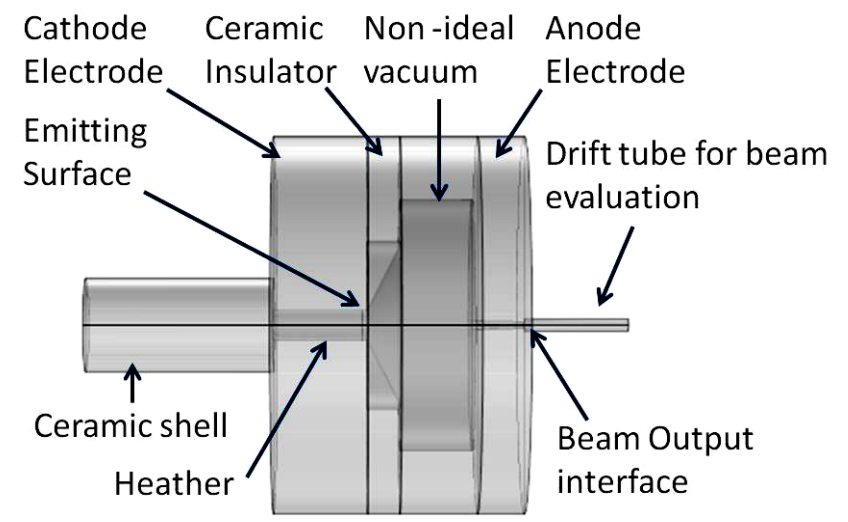

Fig.1. E-Gun Geometry and electric potential distribution.

A Finite Element Method (FEM) simulation, using COMSOL Multiphysics, have been employed to couple TM, ES and CPT analysis by Moving Mesh (MM) dedicated interface and storing temperature information. MM computation moved the mesh in function of the displacement computed by the TM analysis. Subsequently, ES and CPT 
computations have been executed on the new meshes and by receiving the temperatures evaluated by the TM study [7].

A schematization of the computational strategy is represented in Fig. 2. For the proposed device, a directly heated Barium on Tungsten dispenser cathode has been employed with a corrected Richardson constant $A g=1.5 \cdot 10^{3} \mathrm{Am}^{-2} \mathrm{~K}^{-2}$, work function $W=1.56 \mathrm{eV}$ [9] and a surface area of $\mathrm{S}=1.37 \mathrm{~mm}^{2}$ held at the temperature of $T=950^{\circ} \mathrm{C}$. By applying a cathode voltage of $-10 \mathrm{kV}$, the emitted thermionic current is $I=16 \mathrm{~mA}$, derivable from Schottky law shown in Fig. 2, where $e$ is the electron charge, $K_{B}$ the Boltzmann's constant, $E$ the electric field and $J$ the current density [10].

$$
\begin{aligned}
& \text { Multiphysics Thermo-Mechanical Computation }
\end{aligned}
$$

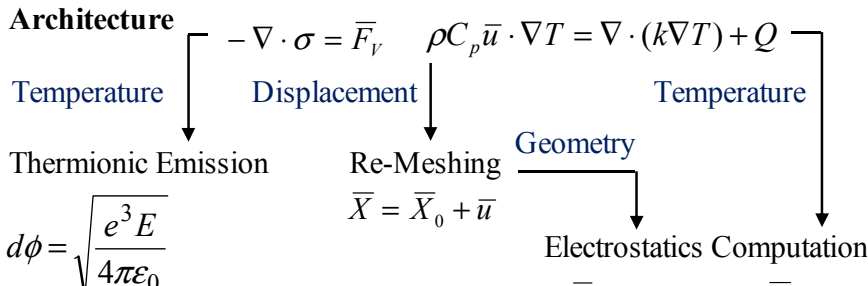

$$
\begin{aligned}
& J=A_{g} T^{2} e^{-\frac{\phi-d \phi}{k_{B} T} \underset{\text { Electric Current }}{\stackrel{\text { Electric Field }}{\longrightarrow}} \begin{array}{c}
\bar{E}=-\nabla V \\
\text { Electric } \\
\text { Field }
\end{array} \downarrow \uparrow \begin{array}{c}
\text { Space } \\
\text { Charge }
\end{array}} \\
& \text { Charged Particle Tracing } \\
& \text { Post-processing } \longleftarrow \text { Beam Dynamics } \frac{\partial\left(m_{p} \bar{v}\right)}{\partial t}=\bar{F}_{t}
\end{aligned}
$$

Fig.2. Logical diagram of the Multiphysics architecture for the computation of the beam dynamics in thermo-mechanical operation.

The aim of this paper is to provide the needed knowledge for developing numerical models which foresee the global behavior of this kind of devices while operating. To clearly show the deterministic consequences of thermo-mechanical effects over the beam dynamics, in this model, the complexity of particle release has been reduced to the least possible, for example by simulating for a little number of particles. By adopting this approach, the alteration of the particle distribution can be evidently shown.

\section{A. Thermo-mechanical Analysis}

The TM analysis is performed by coupling a thermodynamic computation with a structural mechanics simulation, in order to solve the system of steady state stress and heat equations shown in Fig.2 [7]. The value is the stress and $\bar{F}_{V}$ is the force per unit volume; is the density, $C_{p}$ the heat capacity at constant pressure, and $k$ the thermal conductivity of the material. $T$ is the temperature computed on the surfaces, $Q$ is the heat source, $\bar{u}$ is the spatial displacement vector. The net inward heat flux stemming from cathode surface to non ideal vacuum ambient radiation has been computed by solving the equation of the body radiation in the steady state (1) [11].

$$
-\bar{n} \cdot(-k \nabla T)=\varepsilon K_{B}\left(T_{a m b}^{4}-T^{4}\right)
$$

where $T_{a m b}$ is the ambient temperature of the inner volume, is the surface emissivity of the material and $\bar{n}$ the external normal to the surface. At the centre of the cathode structure, the emitting surface is placed. It consists of a constant temperature surface made by Tungsten. At the design temperature $T$, a tungsten alloy emissivity of 0.2 has been assumed as a reasonable value.

The external base surface of the anode, which encircles the beam output interface, is locked to rigid structures in order to support the device. Thus, such external surface represents a mechanical fixed constraint. Gravity acceleration has been also considered, it is directed along the symmetry axis.

A copper heat sinker with a thickness of $5 \mathrm{~mm}$ wraps the lateral surface of the anode and, in order to reduce meshing, it has been modeled as high thermally conductive layer with such specified thickness.

The external environment temperature is $T_{e x t}=25^{\circ} \mathrm{C}$, consistently with a typical environment temperature condition. Boundary conditions on the external walls are used to compute the inward heat flux, with a heat transfer coefficient to the external environment of $h=5 \mathrm{Wm}^{-2} \mathrm{~K}^{-1}$.

The heat transfer mechanism between the gun and the external environment, which surrounds the electron gun, is regulated by the convective heat flux. Such energy exchange has been computed by solving the inward heat flux equation in the steady state (2) [11]:

$$
-\bar{n} \cdot(-k \nabla T)=h\left(T_{e x t}-T\right)
$$

Since temperatures are dependent to the displacements radiation and external temperature, all the thermo-mechanical computations are solved simultaneously.

\section{B. Beam Dynamics}

The ES analysis is employed to solve the system of Electrostatics equations shown in fig. 2 [7], where ${ }_{v}$ is the volumetric charge density, $\bar{D}$ the dielectric displacement vector and $V$ the voltage. Since the anode is grounded, its electric potential is set to zero and the cathode electric

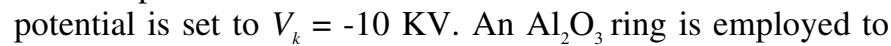
seal the vacuum region, while separating anode to cathode. Since the proposed device would reach a particle energy of 10 $\mathrm{keV}$, expecting a final velocity in the order of $10^{7} \mathrm{~m} / \mathrm{s}$, relativistic effects could be neglected.

The CPT analysis is employed to calculate the particle trajectories considering the ES computation, by solving the CPT equations shown in Fig.2 [7]. The values $m_{p}$ and $\bar{v}$ are respectively the particle mass and velocity, $\bar{F}_{t}$ the total force experienced by the particle.

In order to better evaluate the deterministic effects due to the cathode heating over the whole particle distribution and to clearly follow the single particle trajectories, we have simulated for the minimum reasonably possible number of particles.

However, a statistical distribution is employed to represent the initial velocity $V_{o}$ of thermally excited charges when released from the cathode. The interactions, involving the high energy electrons in the tail of the Fermi-Dirac density of states, allows its replacement with the classical Maxwell- 
Boltzmann distribution [12]. The mean squared velocity in any one dimension can be assumed as (3) [13]:

$V_{R M S}=\sqrt{\frac{K_{B} T}{m_{0}}}$

By comparing the Maxwell-Boltzmann and the Normal distributions has been possible to represent the stochastic behavior by releasing particles with a random initial velocity, following a Normal distribution with specific assumptions [14]: Since electrons does not have preferred direction, a null mean value has been used. The standard deviation has been set equal to $V_{R M S}$ that is, at the design temperature, $V_{R M S}=1.36 \cdot 10^{5}$ $\mathrm{ms}^{-1}$.

Since the cathode current density is constant in time and on the cathode surface, the charge release is represented by a short pulse sequence with initial null value [7-8]. In order to decrease computational costs, the number of particles per release $N$ has been decreased to a thousand times lower integer $N^{l}=244$ and a charge multiplier factor $n=N / N^{l}=2 \cdot 10^{3}$ has been added in order to respect the space charge effect for the design thermionic current $I$.

An opportune condition has been inserted to add the Coulomb interaction forces between particles to the total force.

Electrical breakdown distance in $\mathrm{mm}, d_{m m}$, between anode and cathode can be estimated by the Keller's experimental formula for a certain voltage in $\mathrm{kV}, V_{k V}$ (4) [12]:

$d_{m m}=1.4 \cdot 10^{-2} \cdot V_{k V} \frac{3}{2}$

The smaller distance between electrodes subjected to the maximum voltage is $d_{s}=1.2 \mathrm{~mm}$. Since this distance is higher than the breakdown distance for $10 \mathrm{kV} d_{m m}=0.44 \mathrm{~mm}$, electrical breakdowns can't occur.

\section{COMPUTATIONAL OUTPUTS}

\section{A. Thermo-mechanica Features}

In order to underline the deformation, stress and displacement are been plotted with a magnified scale, so that the deformation scale has been increased. In the following figures, black outlines represent the original conformation, and the stained volume represents the deformed structure.

By imposing the design temperature, the global temperature distribution over the whole device walls has been computed. This result is shown in Fig. 3. Such temperature has induced a maximum stress of about $1.8 \mathrm{GNm}^{-2}$ located at the interface between tungsten and the ceramic shell of the heater and a maximum total displacement, located on backward cathode surface, of about $4.0 \mu \mathrm{m}$ as shown in Fig. 4. These values are critical respect to the e-Gun dimensions, so a considerable alteration of the e-Gun beam forming properties might occur.

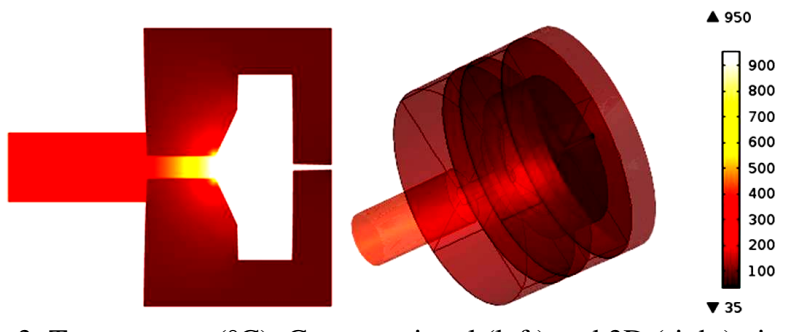

Fig.3. Temperature $\left({ }^{\circ} \mathrm{C}\right)$ : Cross sectional (left) and 3D (right) views.
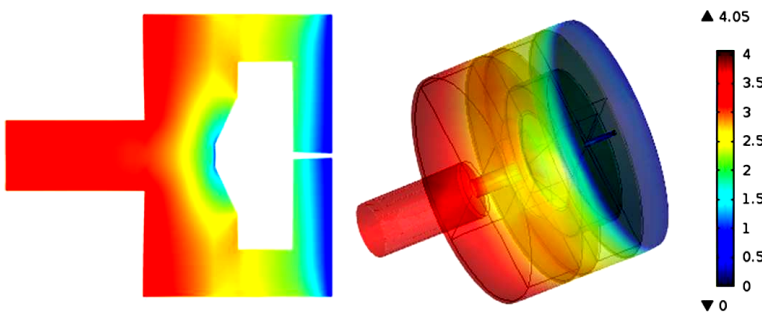

Fig.4. Displacement $(\mu \mathrm{m})$ : Side transparent (left) and 3D views.

\section{B. Electrostatic Fields and Particle Tracing}

The resulting maximum electric fields are: In cold conditions: $E_{M a x}=16.6 \mathrm{MVm}^{-1}$, and in thermo-mechanical operative conditions: $E_{\text {Max }}=19.2 \mathrm{MVm}^{-1}$, due to the evident spatial deformation over the lateral interface. These results are reported in fig. 5 .
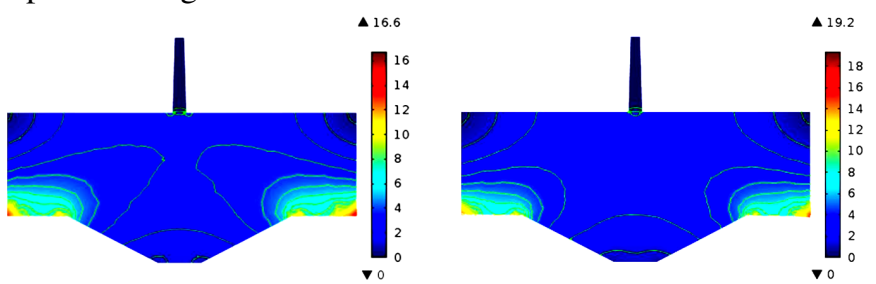

Fig.5. Electric Field (MV/m): Cold (left) and working conditions (right).

\section{Particle Energy and Beam Distribution}

The thermo-mechanical alteration of the e-Gun shape and surface losses contribute to decrease the maximum particle velocity magnitude from $v_{\text {cold }}=5.92 \cdot 10^{7} \mathrm{~ms}^{-1}$ in cold conditions, to $v_{\text {hot }}=5.85 \cdot 10^{7} \mathrm{~ms}^{-1}$ in thermo-mechanical operative conditions. The resulting maximum energies reached by the particle are: in cold conditions $W_{\text {Max }}=9.92 \mathrm{keV}$ and in thermomechanical operation $W_{\text {Max }}=9.89 \mathrm{keV}$, as reported in Fig 6. In thermal stressed conditions, a displacement of the beam trajectory and a de-focalization effect can be noted, due to the deformations of the beam forming electrodes.

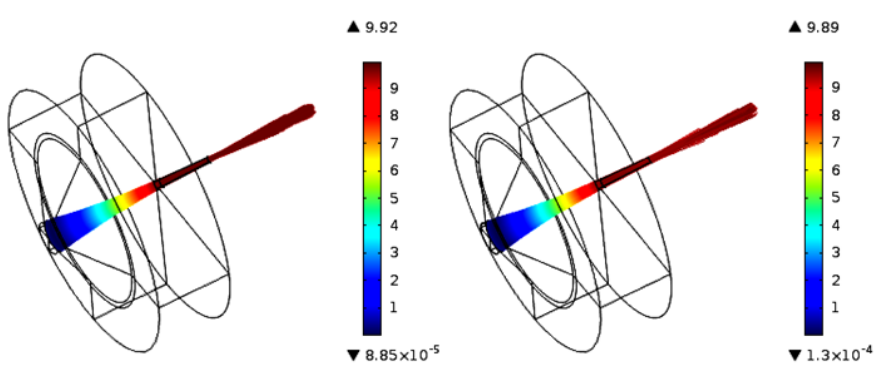

Fig.6. Particle trajectories and energy (keV) in cold conditions (left) and in thermo-mechanical conditions (right). 
The particle spot has been described by using Poincare maps, depicted in the left and right images of figure 7, respectively for cold and thermo-mechanical operative condition. In these figures, green points indicate the distribution wherewith electrons are released from the emitting surface, at $\mathrm{z}=0 \mathrm{~mm}$ and grey points at $\mathrm{z}=0.2 \mathrm{~mm}$. Blue points represent the particle distribution at the end of the cathode, at $\mathrm{z}=1.24 \mathrm{~mm}$. Red points refers to the beam waist at $\mathrm{z}=6.4 \mathrm{~mm}$, $0.4 \mathrm{~mm}$ from the output interface of the anode. These plots given the evidence of the different beam waist distributions. In thermo-mechanical conditions, particle dislocation at the same instant is different to that in cold conditions and the beam waist have a larger surface. In figure 8 and 9 we can observe, respectively the longitudinal distribution in cold and in thermo-mechanical operating conditions. In the upper images, a main representation of the trajectories is depicted, while in the lower images the detail of the region close to the beam waist is reported. By comparing such images, the alteration of the particle distribution is evident when the e-Gun experiences the cathode heating with respect to the cold condition, which neglects this aspect.

The beam waist has a radius $r=75 \mu \mathrm{m}$ in cold conditions and $\mathrm{r}=100 \mu \mathrm{m}$ in thermo-mechanical conditions. By referring to the emitting surface, in thermo-mechanical condition, the proposed e-Gun shows a beam area compression factor of 34, without employing external magnets.
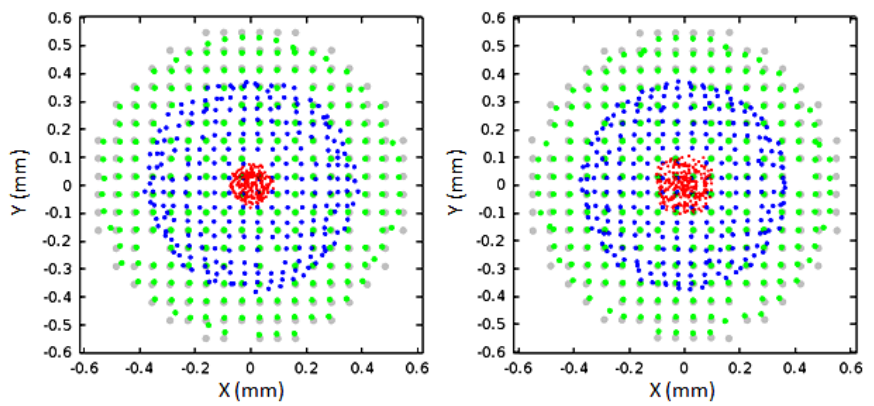

Fig.7. Spot cross sectional position in cold conditions (left) and in thermomechanical operation (right).

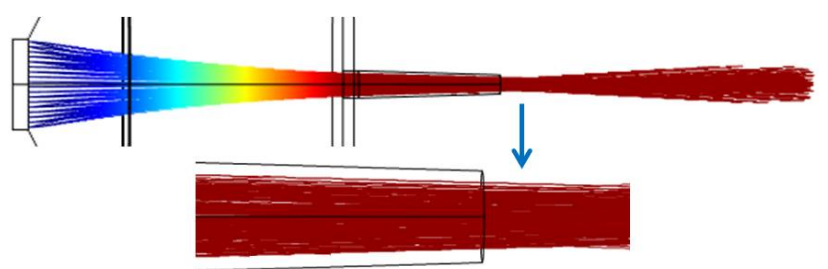

Figure 8. Longitudinal view of particle trajectories in cold conditions: Main distribution (upper) and close to the beam waist (lower).

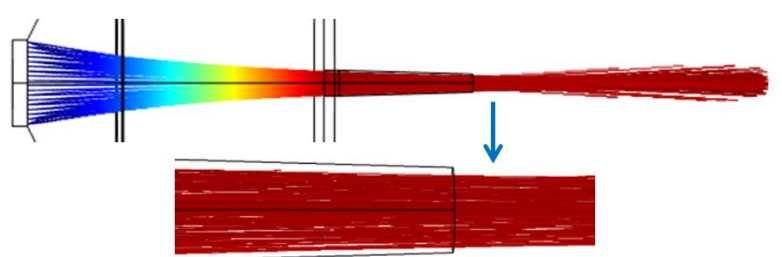

Figure 9. Longitudinal view of particle trajectories in thermo-mechanical operation: Main distribution (upper) and close to the beam waist (lower).

\section{CONCLUSIONS}

An electron gun suitable for Submillimeter wave and spatial environment applications has been designed through a multiphysics modeling approach. Electric field and particle tracing have been computed to evaluate the thermomechanical effects induced by the cathode heating over the beam dynamics. Several strategies have been adopted to obtain a simple model, which shows clearly the investigated mechanisms. The Beam distribution of the analyzed device has been documented, in cold and in thermo-mechanical operative conditions.

This device, can produce a $16 \mathrm{~mA}$ electron beam with 10 $\mathrm{keV}$ energy. When the e-Gun experiences the cathode heating, the beam waist remains located at the anode output interface and changes from $75 \mu \mathrm{m}$ to $100 \mu \mathrm{m}$ of radius. This is still an acceptable value for this purpose. The proposed Multiphysics approach allows for the prediction of the operative behavior of a thermionic e-Gun where the small dimensions makes the operation extremely critical while the cathode heating effects are present. According to this study, the appropriate materials and shapes can be chosen.

\section{REFERENCES}

[1] J. R. Pierce, Theory and Design of Electron Beams, D. Van Nostrand Company, Canada, The Bell Telephone Laboratories Series, 1949.

[2] F. Hinode et al.: "Present Status of Thermionic RF-Gun for Terahertz Source Project at Tohoku University" proc. of FEL 2012, Nara, Japan, pp. 137-139.

[3] F. Hinode et al.: "Development of a Thermionic Rf Gun For Coherent THz Source at Tohoku University", Proc. of IPAC 2010, Kyoto, Japan, pp.1731-1733.

[4] J. A. Gaskin et Al., Simulation and Characterization of a Miniaturized Scanning Electron Microscope, IEEE Aerospace Conference, 2011.

[5] J. A. Gaskin et Al., "Miniature Scanning Electron Microscope for InSitu Planetary Studies: Electron Gun Development", Proc. of 40th Lunar and Planetary Science Conference, 2009.

[6] J. Skevington and T. Schneider "NASA USRP - Intern Final Report Installation and Characterization of Charged Particle Sources for Space Environmental Effects Testing", NASA Marshall Space Flight Center, Huntsville, 2010, pp.1-10.

[7] A. Leggieri, D. Passi, F. Di Paolo, "Multiphysics Modeling Based Design of a Key-Holes Magnetron," Proc. of IEEE International Conference on Numerical Electromagnetic Modeling and Optimization, Pavia, Italia, 2014.

[8] Leggieri A., Ulisse G., Di Paolo F., Brunetti F., Di Carlo A., "Particle tracing simulation of a vacuum electron gun for THz application", in Proc. of IEEE Millimeter Waves and THz Technology.

[9] C. J. Smithells: "Electron emission" in Metals Reference Book, Vol III, Butterworths, London, 1967. Chap.18 p.2.

[10] J.M.D. Kowalczyk, M.R. Hadmack and J.M.J. Madey: "Laser Cooling to Counteract Back-Bombardment Heating in Microwave Thermionic Electron Guns", Proc. of FEL2013, New York, NY, USA, pp.79-81.

[11] J. M. Coulson and J. F. Richardson, "Heat transfer by convection" in Chemical Engineering, Vol. 1, $6^{\text {th }}$ Ed., Butterworth Heinemann, Oxford, UK, 1999, pp.414-471.

[12] M. Reiser, "Source of Charged Particles" in Theory and Design of Charged Particle Beams, WILEY-VCH Verlag GmbH \& Co., Weinheim, 2004, p 13.

[13] D. H. Dowell, "Electron Emission And Cathode Emittance" in High Brightness Electron Injectors for Light Sources, U.S. Particle Accelerator School, Course Materials, 2010, pp.4-5.

[14] Sheskin, D. J.; "The Normal Distribution" in Handbook of Parametric and Nonparametric Statistical Procedures, $3^{\text {rd }}$ ed., CRC Press, Boca Raton, 2003, pp. 42-44. 